\title{
Can surgical diagnosis of "early" gastric cancer and lymph node metastasis be accurate?
}

\author{
Takaki Yoshikawa, Naoki Ishiwa, Soichiro Morinaga, Yoshikazu Noguchi, and Yuji Yamamoto \\ Department of Surgery, Yokohama City Kowan Hospital, 3-2-3 Shinyamashita, Naka-ku, Yokohama 231-0801, Japan
}

\begin{abstract}
Background. The objective of this study was to evaluate the accuracy of the intraoperative assessment of early gastric cancer (EGC) and lymph node status.

Methods. A total of 280 patients underwent gastrectomy for primary gastric cancer at the Department of Surgery, Yokohama City Kowan Hospital, from 1994 to 2002. Of these 280 patients, 140 were diagnosed as having EGC during surgery (sT1), while the rest were diagnosed with advanced disease (sT2-T4). Of the former 140 patients, 136 patients, who underwent curative resection with D1 or D2 lymphadenectomy, were further investigated for the assessment of lymph node status.

Results. The sensitivity, specificity, and accuracy of the surgical diagnosis of EGC were $96.4 \%$ (135 of 140$), 90.0 \%$ (126 of 140 ), and $93.2 \%$ (261 of 280$)$, respectively, while these values for the assessment of lymph node metastases were $55.6 \%$ (5 of 9), $94.5 \%$ (120 of 127), and 91.9\% (125 of 136), respectively. In a further analysis in which patients with tumors of macroscopically depressed type and undifferentiated histology were excluded, all patients staged as sT1sNo had no lymph node metastases pathologically.

Conclusion. Although the depth of tumor invasion can be correctly assessed intraoperatively, it is difficult to be precise regarding the presence of nodal metastases. One practical solution to this problem might be to employ limited surgery for those patients with macroscopically elevated and histologically differentiated tumors.
\end{abstract}

Key words Early gastric carcinoma - Lymph node dissection · Surgical diagnosis

\section{Introduction}

Early gastric cancer (EGC) is defined as that in which invasion is confined to either the mucosa or submucosa,

Offprint requests to: $\mathrm{T}$. Yoshikawa

Received: July 28, 2003 / Accepted: October 20, 2003 regardless of regional lymph node metastasis [1]. As a result of increased diagnostic accuracy and the wider employment of mass surveys, the proportion of EGC among gastric cancers has steadily increased, not only in Japan but also in some Western countries [2-4]. A survival rate of greater than $90 \%$ has been repeatedly documented for patients with EGC treated with radical surgery [5-10].

A treatment widely employed for EGC in Japan and in some European countries has been total or subtotal gastrectomy with D2 lymphadenectomy [11]. However, a new trend in Japan is to customize surgery, depending on the stage of disease [12], because the involvement of lymph nodes is relatively infrequent and metastasis to the level 2 lymph nodes is rare [8,10]. Many investigators have proposed indications for limited dissection based on the clinicopathological analyses of resected specimens [13-17]. However, surgeons have to determine the type of dissection during surgery before the pathology reports arrive. How accurate can our diagnoses be?

Recently, the Japanese Gastric Cancer Association published guidelines for treating gastric cancer [18]. According to these guidelines, the choice of lymph node dissection is determined based on the pre- and intraoperative diagnoses of the depth of invasion (sT) and level of lymph node metastasis ( $\mathrm{sN}$ ). This means that the accuracy of the final diagnosis all depends on the surgeons. Evaluation of these data at each institution should be mandatory, as it is for sentinel nodes in breast cancer. In this study, we attempted to evaluate the accuracy of the intraoperative assessment of EGC and lymph node status.

\section{Patients and methods}

From 1994 to 2002, 280 patients underwent gastrectomy for primary gastric cancer at the Department of Sur- 
gery, Yokohama City Kowan Hospital. The depth of invasion and lymph node metastases were evaluated preoperatively by computed tomography (CT) scans, fiberscopic examinations, and upper gastrointestinal series, and finally assessed macroscopically during the operation (surgical findings) and microscopically after the resection (pathological findings). The macroscopic diagnosis of depth of invasion was made from such findings as changes in the serosal surface, indurations, or mobility in the gastric wall. The status of lymph nodes was judged from the size of nodes and consistency by palpation. Depth of invasion and the presence of lymph node metastases were assessed visually and by palpation just after the operation, based on the macroscopic investigation of the resected specimens, and the findings were recorded according to the General rules for gastric cancer study [1]. Of the 280 patients, 140 were diagnosed as having EGC during surgery (sT1), while the rest were diagnosed with advanced disease (sT2-T4). Of the former 140 patients, 136 patients (a group with D1 dissection $[n=35)$ and a group with D2 lymph node dissection $[n=101])$, were further investigated for this study; the remaining 4 patients were excluded because they underwent partial resection of the stomach without systemic lymph node dissection. Limited D1 dissection was employed for patients with severe complications or those with small, clinically diagnosed mucosal tumors that were unlikely to have lymph node metastases.

The surgical diagnosis of EGC was evaluated by determining the sensitivity (true positive rate; i.e., the number of lesions surgically diagnosed and pathologically confirmed as EGC divided by the number of surgically diagnosed EGCs), the specificity (true negative), and the accuracy (the number of lesions whose surgical and pathological diagnoses were in accordance divided by the total number of lesions). The accuracy of the surgical diagnosis of lymph node metastases was evaluated similarly. The $\chi^{2}$ method and unpaired Student's $t$-test were used for comparisons between two groups. Logistic regression was used for multivariate analyses.

\section{Results}

\section{Surgical diagnosis of EGC}

Comparisons of surgical and pathological diagnoses are shown in Table 1 . The sensitivity, specificity, and accuracy of the surgical diagnosis of EGC were $96.4 \%$ (135 of 140 ), $90.0 \%$ (126 of 140), and $93.2 \%$ (261 of 280 ), respectively. In five patients with a false-positive sT1, three tumors had invaded the proper muscle, without lymph node metastases ( $\mathrm{mp}$ and $\mathrm{pN} 0$ ), and two had invaded the subserosa, one with metastases to the level 1 nodes (ss and pN1), and the other to the level 2 nodes (ss and pN2). Macroscopic type, histological type, and maximal diameter obtained before surgery were compared between patients with a true-positive (sT1pT1) diagnosis and those with a false-positive (sT1pT2-4) diagnosis (Table 2). Although there was no significant difference between the two groups, all five patients with a false-positive diagnosis had macroscopically depressed type tumors. By Cox's regression analysis, none of the above three factors were significant predictors for the diagnosis of tumor depth.

\section{Surgical diagnosis of lymph node metastases in sT1}

The 136 patients who were diagnosed as having EGC (sT1) underwent curative resection with D2 or D1 lymph node dissection, and 127 of these patients were diagnosed as having no lymph node metastasis during surgery ( $\mathrm{sN} 0$ ), while the remaining 9 patients were diag-

Table 1. Surgical diagnosis of early gastric cancer

\begin{tabular}{lccc}
\hline & \multicolumn{2}{c}{ Pathological diagnosis } & \\
\cline { 2 - 3 } Surgical diagnosis & pT1 (m/sm) & pT2 $(\mathrm{mp} / \mathrm{ss})$ & Total \\
\hline sT1 & $135(81 / 54)$ & $5(3 / 2)$ & 140 \\
sT2-T4 & $14(1 / 13)$ & 126 & 140 \\
\hline
\end{tabular}

$\mathrm{m}$, mucosa; sm, submucosa; mp, proper muscle; ss, subserosa

Table 2. Clinicopathological characteristics of patients with true (sT1pT1)- and false (sT1pT2-4)-positive diagnoses

\begin{tabular}{lccc}
\hline & \multicolumn{2}{c}{ Diagnosis } & \\
\cline { 2 - 3 } Tumor & $\begin{array}{c}\text { True positive } \\
(\text { sT1pT1) }\end{array}$ & $\begin{array}{c}\text { False positive } \\
(\text { sT1pT2-4) }\end{array}$ & $P$ value \\
\hline Macroscopic type & $93 / 42$ & $5 / 0$ & NS \\
$\begin{array}{l}\text { Depressed/flat or elevated } \\
\text { Histological type }\end{array}$ & $109 / 26$ & $4 / 1$ & NS \\
$\begin{array}{l}\text { Differentiated/undifferentiated } \\
\text { Maximal diameter (mm) }\end{array}$ & $25.7 \pm 22.1$ & $40.8 \pm 30.7$ & NS \\
\hline NS, not significant & & &
\end{tabular}


nosed with metastases to the level 1 nodes (sN1). None was diagnosed to have metastases to the level 2 nodes (sN2). The surgical and pathological diagnoses are shown in Table 3. The sensitivity, specificity, and accuracy of the surgical diagnosis of lymph node metastases were $55.6 \%$ (5 of 9), $94.5 \%$ (120 of 127), and $91.9 \%$ (125 of 136), respectively. Table 4 shows the data for macroscopic type, histological type, and maximal diameter (all of which details were obtained before surgery) in patients with true-negative ( $\mathrm{sN} 0 \mathrm{pN} 0)$ and false-negative (sN0pN1-3) diagnoses. Only histological type was significantly different between the two groups. By Cox's regression analysis, histological type was a significant predictor of a false-negative result $(P=$ 0.026; hazard ratio, 6.53).

\section{Analysis of data in selected patients (Table 5)}

In the above analyses of the surgical diagnosis of EGC, it became clear that all patients with a false-positive diagnosis had macroscopically depressed-type tumors and that a tumor with undifferentiated histology was a significant predictor of underestimation. Excluding patients with these two factors of macroscopically depressed type and undifferentiated histology, we examined the clinical value of the surgical diagnosis of lymph node metastases. Twenty-eight patients were eligible for this analysis and were selected from the 136 patients who were diagnosed surgically as having EGC (sT1). Of the 28 patients, 25 were diagnosed as having $\mathrm{sN} 0$ and 3 as having sN1. The sensitivity, specificity, and

Table 3. Surgical diagnosis of lymph node metastases

\begin{tabular}{lrcr}
\hline & \multicolumn{2}{c}{ Pathological diagnosis } & \\
\cline { 2 - 3 } Surgical diagnosis & $\mathrm{pN} 0$ & $\mathrm{pN}+(\mathrm{pN} 1 / \mathrm{pN} 2)$ & Total \\
\hline sN0 & 120 & $7(4 / 3)$ & 127 \\
sN1 & 4 & $5(3 / 2)$ & 9 \\
sN2 & 0 & 0 & 0 \\
\hline
\end{tabular}

accuracy for the diagnosis of lymph node metastases were $66.7 \%(2 / 3), 100 \%(25 / 25)$, and $96.4 \%$ (27/28). All patients diagnosed as having sT1sN0 with macroscopically elevated and histologically differentiated tumors had no lymph node metastases pathologically.

\section{Discussion}

According to the guidelines of the Japanese Gastric Cancer Association, the optimal lymph node dissection for gastric cancer should be determined based on the surgical diagnosis of the depth of invasion (sT) and the extent of lymph node metastases (sN) [18]. In EGC, for example, sT1sN0 should be treated by limited D1 + alpha and D1 + beta dissection ("D1 + alpha" dissection includes perigastric nodes and no. 7 nodes for tumors located in the middle or upper third of the stomach, and no. 7 and no. 8a nodes for tumors located in the lower third of the stomach, while "D1 + beta" dissection denotes no. 7, no. 8 a, and no. 9 node dissection besides perigastric nodes); sT1sN1 should be treated by limited D1 + beta or D2 dissection; and sT1sN2 should be treated by $\mathrm{D} 2$ dissection. To enable these decisions to be made, surgical diagnosis of the stage has to be accurate. However, the accuracy of pre- and intraoperative diagnosis of EGC and lymph node metastases has not been reported so far.

The surgical diagnosis of early disease was documented to be highly accurate, with high sensitivity and specificity, in the present study. The surgical diagnosis

Table 5. Accuracy of surgical diagnosis of node metastasis in patients with differentiated histology and macroscopically elevated-type tumors

\begin{tabular}{lcc}
\hline & pN0 & pN1-pN2 \\
\hline sT1sN0 $^{a}$ & 25 & 0 \\
sT1sN1 $^{b}$ & 1 & 2 \\
\hline
\end{tabular}

a The ratio of $\mathrm{m}$ to $\mathrm{sm}$ was $18: 7$

${ }^{\mathrm{b}}$ Includes two patients with sm and one with ss

Table 4. Clinicopathological characteristics of patients with true (sN0pN0)- and false (sN0pN1-3)-negative diagnoses

\begin{tabular}{|c|c|c|c|}
\hline \multirow[b]{2}{*}{ Tumor } & \multicolumn{2}{|c|}{ Diagnosis } & \multirow[b]{2}{*}{$P$ value } \\
\hline & $\begin{array}{l}\text { True negative } \\
\text { (sN0pN0) }\end{array}$ & $\begin{array}{l}\text { False negative } \\
\text { (sN0pN1-2) }\end{array}$ & \\
\hline $\begin{array}{l}\text { Macroscopic type } \\
\text { Depressed/flat or elevated }\end{array}$ & $90 / 30$ & $6 / 1$ & NS \\
\hline $\begin{array}{l}\text { Histological type } \\
\text { Differentiated/undifferentiated } \\
\text { Maximal diameter }(\mathrm{mm})\end{array}$ & $\begin{array}{c}96 / 19 \\
24.3 \pm 21.8\end{array}$ & $\begin{array}{c}3 / 4 \\
32.6 \pm 27.3\end{array}$ & $\begin{array}{c}0.023 \\
\text { NS }\end{array}$ \\
\hline
\end{tabular}


of depth of tumor invasion was made intraoperatively not only by changes in the serosal surface but also by the mobility of the tumor on the muscle layer, in addition to the preoperative diagnosis made by endoscopy and upper gastrointestinal series. These diagnoses are highly dependent on the experience of surgeons.

Of our patients diagnosed as having "early" disease or sT1, the diagnoses in five patients were found to be false-positive (sT1pT2-4). Although there were no significant differences in the pathological factors examined between the patients with a correct diagnosis (sT1pT1) and those with a false-positive one (sT1pT1-4), all macroscopically elevated and flat-type tumors were correctly diagnosed as pT1. However, two of the five patients with a false-positive diagnosis patients had lymph node metastases. Thus, we should be more cautious in determining the depth of invasion of depressedtype tumors.

Considering the intraoperative assessment of lymph node metastases, it is accepted that it is not safe to diagnose lymph node metastases by size and palpation. However, there is no accurate way to diagnoses lymph node status. The new method of sentinel node biopsy has not yet been shown to be conclusive. In some tertiary hospitals, the pathological status of the nodes is checked by the analysis of frozen sections when malignant involvement is highly suspected, especially in far advanced disease. However, this method has not been accepted as a community standard for early disease. In many general hospitals, lymph node status in EGC is evaluated by the size and consistency of the nodes. Our objective was, therefore, to re-assess the accuracy of the standard methods and to elucidate the subset of the population in whom these routine gross methods can be safely employed.

The incidence of lymph node metastasis in EGC has been reported to be less than $20 \%[8,10]$. The high specificity but low sensitivity in the surgical diagnosis of lymph node metastases in the present study indicates the difficulty of diagnosing the presence of lymph node metastases intraoperatively. Other authors have also demonstrated the low sensitivity of the surgical diagnosis of nodal involvement [19-22]. Usually, lymph node metastases were evaluated by the size and consistency of the nodes during surgery. However, increased size does not necessarily indicate the presence of metastasis [23]. Also, it is very rare to find enlarged lymph nodes in patients with an early stage disease. In the present series, 35 patients underwent D1 dissection and the remaining patients had a D2 dissection. It is possible that patients who underwent limited dissection had tumors invading lymph nodes at $\mathrm{N} 2$ stations which were not dissected. Therefore, in this study, the parameters we used to assess surgical diagnosis may have led to slight underestimation of the sensitivity.
In employing limited surgery in patients with EGC, the major problems are the underestimation of the depth of tumor invasion and the extent of lymph node metastases, because some of these patients could be cured by radical D2 lymphadenectomy. In our study, there were seven patients whose nodal status was underestimated (N0pN1-3). To examine whether these patients could have been predicted, we analyzed the macroscopic type, histological type, and maximal diameter of tumors by univariate and multivariate analyses comparing those with sN0pN0 (correctly diagnosed) and those with sN0pN1-3 (underestimated). Of the three factors examined, histological type was found to be a significant factor. These data suggest that the correct diagnosis of nodal metastasis during surgery is more difficult in undifferentiated than in differentiated carcinomas.

When patients with tumors of macroscopically depressed type and undifferentiated histology were excluded from our analysis, we found that all patients with sN0 had no lymph node metastasis pathologically. Therefore, limited nodal dissection could be safely performed in these selected patients.

In conclusion, the customization of surgery for EGC has been advocated. The low incidence of nodal metastasis and high survival rate are the basis for this recommendation. Although the depth of tumor invasion can be correctly assessed surgically, the presence of nodal metastases is hard to detect. One practical solution to this problem may be to employ limited surgery for those patients with macroscopically elevated and histologically differentiated tumors.

\section{References}

1. Japanese Gastric Cancer Association. Japanese classification of gastric carcinoma. 2nd English Edition. Gastric Cancer 1998;1:1024.

2. Hochwald SN, Brennan MF, Klimstra DS, Kim S, Karpeh MS. Is lymphadenectomy necessary for early gastric cancer? Ann Surg Oncol 1999;6:664-70.

3. Sue-Ling HM, Martin I, Griffith J, Ward DC, Quirke P, Dixon MF, et al. Early gastric cancer: 46 cases treated in one surgical department. Gut 1992;33:1318-22.

4. Noguchi Y, Yoshikawa T, Tsuburaya A, Motohashi H, Karpeh MS, Brennan MF. Is gastric carcinoma different between Japan and the United States? Cancer 2000;89:2237-46.

5. Itoh H, Oohata Y, Nakamura K, Nagata T, Mibu R, Nakayama F. Complete 10-year postgastrectomy follow-up of early gastric cancer. Am J Surg 1989;158:14-6.

6. Ohta H, Noguchi Y, Takagi K, Nishi M, Kajitani T, Kato Y. Early gastric carcinoma with special reference to macroscopic classification. Cancer 1987;60:1099-106.

7. Kito T, Yamamura Y, Kobayashi S. Surgical treatment of early gastric cancer. Anticancer Res 1989;8:335-8.

8. Koga S, Kaibara N, Tamura H, Nishidoi H, Kimura O. Cause of late postoperative death in patients with early gastric cancer: special reference to recurrence and the incidence of metachronous primary cancer in other organ. Surgery 1984;6:511-16. 
9. Kitaoka T, Yoshikawa K, Hirota T, Itabashi M. Surgical treatment of early gastric cancer. Jpn J Clin Oncol 1984;14:28393.

10. Yoshikawa T, Tsuburaya A, Kobayashi O, Sairenji M, Motohashi $\mathrm{H}$, Noguchi Y. Is D2 lymph node dissection necessary for early gastric cancer? Ann Surg Oncol 2002;9:401-5.

11. Noguchi Y, Imada T, Matsumoto A, Coit DG, Brennan MF. Radical surgery for gastric cancer: a review of the Japanese experience. Cancer 1989;64:2053-62.

12. Noguchi Y, Morinaga S, Yamamoto Y, Yoshikawa T. Is there a role for nontraditional resection of early gastric cancer? Surg Oncol Clin N Am 2002;11:387-403.

13. Yoshikawa T, Tsuburaya A, Kobayashi O, Sairenji M, Motohashi $\mathrm{H}$, Noguchi $\mathrm{Y}$. Indications of limited surgery for gastric cancer with submucosal invasion. Analysis of 715 cases with special reference to site of the tumor and level 2 lymph nodes. Hepatogastroenterology 2003;50:1727-30.

14. Kunisaki C, Shimada H, Nomura M, Akiyama H. Appropriate lymph node dissection for early gastric cancer based on lymph node metastases. Surgery 2001;129:153-7.

15. Hiki Y, Shimao H, Mieno H, Sakakibara Y, Kobayashi N, Saigenji K. Modified treatment of early gastric cancer: evaluation of endoscopic treatment of early gastric cancers with respect to treatment indications groups. World J Surg 1999;23:187-93.

16. Tsujitani S, Oka S, Saito H, Kondo A, Ikeguchi M, Maeta M, et al. Less invasive surgery for early gastric cancer based on the low probability of lymph node metastasis. Surgery 1999;125:14854.

17. Kitamura K, Yamaguchi T, Taniguchi H, Hagiwara A, Sawai K, Takahashi T. Analysis of lymph node metastasis in early gastric cancer: rationale of limited surgery. J Surg Oncol 1997;64:42-7.

18. Nakajima T. Gastric cancer treatment guidelines in Japan. Gastric Cancer 2002;5:1-5.

19. Korenaga D, Okuyama T, Orita $\mathrm{H}$, Anai H, Baba H, Maehara $\mathrm{Y}$, et al. Role of intraoperative assessment of lymph node metastasis and serosal invasion in patients with gastric cancer. J Surg Oncol 1994;55:250-4.

20. Sano T, Kobori O, Nagawa H, Muto T. The macroscopic diagnosis of lymph node metastases from early gastric cancer. Surg Today 1994;24:37-9.

21. Perng DS, Jan CM, Wang WM, Chen LT, Su YC, Liu GC, et al. Computed tomography, endoscopic ultrasonography and intraoperative assessment in TN staging of gastric carcinoma. J Formos Med Assoc 1996;95:378-85.

22. Ziegler K, Sanft C, Zimmer T, Zeitz M, Felsenberg D, Stein H, et al. Comparison of computed tomography, endosonography, and intraoperative assessment in TN staging of gastric carcinoma. Gut 1993;34:604-10.

23. Monig SP, Zirbes TK, Schroder W, Baldus SE, Lindemann DG, Dienes HP, et al. Staging of gastric cancer: correlation of lymph node size and metastatic infiltration. AJR Am J Roentgenol 1999; 173:365-7. 